\title{
Antibiotic Prescribing Patterns by Health Care Providers in Pediatric Outpatients: A Prospective Observational Study
}

\author{
Siva Kumar Ramasamy ${ }^{1}$, Prachi Goyal ${ }^{1,{ }^{*}}$ and Nirbhay Mehta ${ }^{1}$ \\ ${ }^{1}$ Mahatma Gandhi Memorial Medical College, Indore, India \\ "Corresponding author: Mahatma Gandhi Memorial Medical College, Indore, India. Email: drprachio9@gmail.com
}

Received 2020 August 28; Revised 2021 January 03; Accepted 2021 March 07.

\begin{abstract}
Objectives: To audit the patterns and quality of antibiotic prescription for children in the outpatient department (OPD).

Methods: This prospective observational study was conducted on the tertiary care outpatient department of a teaching hospital. One thousand prescriptions for children between 1 month and 15 years of age, who attended the outpatient department of a tertiary care teaching hospital between April 2018 and May 2019, were included. The quality of prescriptions was assessed based on 12 pretest parameters. One score was given to each correctly written parameter, and total scores were categorized as poor ( 0 - 4$)$, average ( 5 - 8 ), and $\operatorname{good}(9-12)$. The core prescribing indicators presented by the world health organization (WHO) were used to analyze antibiotic prescribing patterns. The data were analyzed using open-source Epidata software.

Results: Out of 1000 prescriptions, quality was average in 490 (49\%) and poor in 46 (4.6\%) prescriptions. The average number of medicines prescribed per encounter was 3.5 (reference value $<2$ ). The medicines were prescribed by their generic names in $27.3 \%$ of the prescriptions (reference value 100\%). Antibiotics and injections appeared in 65\% (reference value $<30 \%$ ) and $6 \%$ (reference value $<20 \%$ ) of the prescriptions. The ratio of prescription from a list of essential medicines was $15 \%$ (reference value $100 \%$ ). According to the prescriber profile, the rate of prescribing an antibiotic was $63 \%$ by postgraduates in pediatrics, $70 \%$ by MBBS, and $90 \%$ by AYUSH doctors (reference value $<30 \%$ ).

Conclusions: More than half of the prescriptions could not attain a good score. There is room for improving prescription writing practice. Antibiotic prescription by health care providers, especially AYUSH doctors, needs to be restricted given the high number of antibiotics per prescription. This will limit the indiscriminate use of antibiotics and may be a big step towards achieving the antibiotic stewardship goal.
\end{abstract}

Keywords: Outpatient Department Prescription, Prescription Quality, Antibiotic Prescribing Pattern

\section{Background}

Antibiotics inhibit the growth of or destroy microorganisms and have completely changed the present-day practice of medicine. Even lethal infections are readily treatable by new-generation antibiotics that have even improved other medical therapies like cancer chemotherapy and organ transplantation in children (1). Given the widespread therapeutic applications of antibiotics, their usage has now spread everywhere, leading to antibiotic resistance. So, it is critical to consider strict stewardship towards antibiotic use.

The easiest method to investigate prescription patterns is to carry out an audit on prescriptions to recognize commonly used antibiotics and drugs among various populations and identify polypharmacy as a serious emerging threat in the community. Auditing also reveals the use or misuse of medications with special reference to antibiotics
(2). In addition, auditing helps in formulating and modifying treatment guidelines according to the needs of the population under study in order to restrict antibiotic overprescription by physicians and nail antibiotic stewardship. It also helps to provide an evidence-based approach to judicious antimicrobial use (3).

In the present study, we aimed to audit the prescriptions of the referral patients coming to the outpatient pediatrics department of a tertiary care teaching hospital in Indore, wherein prescriptions were analyzed using pre-set parameters.

\section{Objectives}

To audit the patterns and quality of antibiotic prescription for children in the outpatient department (OPD). 


\section{Methods}

This cross-sectional observational study was conducted in a tertiary care teaching hospital in central India from April 2018 to May 2019. The study was approved by the Institutional Ethics Committee, and written informed consent was obtained from participants' parents.

One-thousand prescription sheets of pediatric patients aged 1 month to 15 years attended the outpatient department during the study period were selected. The health care providers writing the prescriptions were unaware of the audit. The first author collected prescription sheets and gathered patients' demographics and drug-related information.

A pre-validated list of 12 essential parameters (Table 1) was used to assess the quality of the prescriptions audited. A pilot study was done to validate the selected parameters and the feasibility of the study, after which the audit was finalized and performed.

\begin{tabular}{lc}
\hline Table 1. The Parameters Used for the Quality Assessment of Prescription Sheets \\
\hline Serial No. & Audit Parameters \\
\hline $\mathbf{1}$ & Patient Name \\
$\mathbf{2}$ & Age \\
\hline $\mathbf{3}$ & Date \\
$\mathbf{4}$ & OPD number \\
$\mathbf{5}$ & Diagnosis \\
$\mathbf{6}$ & Dose of drug \\
\hline $\mathbf{7}$ & Route of drug administration \\
$\mathbf{8}$ & Frequency of drug administration \\
\hline $\mathbf{9}$ & Duration of drug usage \\
\hline $\mathbf{1 0}$ & Generic name \\
\hline $\mathbf{1 1}$ & Legibility \\
\hline $\mathbf{1 2}$ & Signature of the doctor \\
\hline
\end{tabular}

One score was given to each correctly written parameter, delivering a total score of 12 . The quality of prescriptions was categorized as either poor (score 0 - 4), average (score 5 -8), or good (score 9 -12).

The prescribing indicators of the world health organization (WHO) were used to analyze antibiotic prescribing patterns and five important areas regarding the appropriate use of medicines (4). The indicators with their reference values (5-7) included:

- Indicator 1: The average number of medicines prescribed per encounter (ref value $<2$ ).

- Indicator 2: The percentage of medicines prescribed by generic name (ref value $=100 \%$ ).

- Indicator 3: The percentage of encounters with an antibiotic prescribed (ref value $<30 \%$ ).
- Indicator 4: The percentage of encounters with an injection prescribed (ref value $<20 \%$ ).

- Indicator 5: The percentage of medicines prescribed from a list of essential medicines (ref value $=100 \%$ ).

The indicator 3 was used to analyze antibiotic prescription by health care providers using the following formula.

$\%$ encounters $=$ No. of clinical encounters with one or more antibiotics prescribed / Total number or encounters

Based on the qualification of prescribers, all audited prescriptions were categorized based on being ordered by Postgraduates in Pediatrics, MBBS, and AYUSH (an acronym for Ayurveda, Yoga and naturopathy, Unani, Siddha, and Homeopathy) incorporating six health fields, which is practiced in India and some of its neighboring Asian countries.

Data were entered in an excel sheet, and descriptive statistics and open-source Epidata software was used for statistical analyses.

\section{Results}

This prospective observational study was conducted from April 2018 to May 2019. During the study period, a total of 1000 prescription sheets were analyzed. Out of 1000 prescriptions, 830 ( $83 \%$ ) were ordered by postgraduates in pediatrics, 130 (13\%) by MBBS, and 40 (4\%) by AYUSH doctors.

The distribution of audit parameters has been shown in Table 2. Out of 1000 prescriptions, 490 (49\%), 464 (46.4\%), and 46 (4.6\%) had average, good, and poor qualities, respectively (Figure 1 ).

\begin{tabular}{lcc}
\hline Table 2. The Distribution Patterns of Audit Parameters & a & \\
\hline Audit Parameters & Yes & No \\
\hline The patient's name & 98.1 & 1.9 \\
\hline Age & 88 & 12 \\
\hline Date & 87.2 & 12.8 \\
\hline Opd number & 58.1 & 41.9 \\
\hline Diagnosis & 27 & 73 \\
\hline Dose & 89 & 11 \\
\hline Route & 68 & 32 \\
\hline Frequency of drug & 90.3 & 9.7 \\
\hline Duration of drug & 86.4 & 13.6 \\
\hline Legibility & 54.2 & 45.8 \\
\hline Generic name & 29.3 & 70.7 \\
\hline $\begin{array}{l}\text { Signature of the } \\
\text { doctor }\end{array}$ & 62 & 38 \\
\hline a Values are expressed as percentage. & \\
\hline
\end{tabular}

J Compr Ped. 2021;12(2):e108953. 
Percentage Distribution of Quality Score

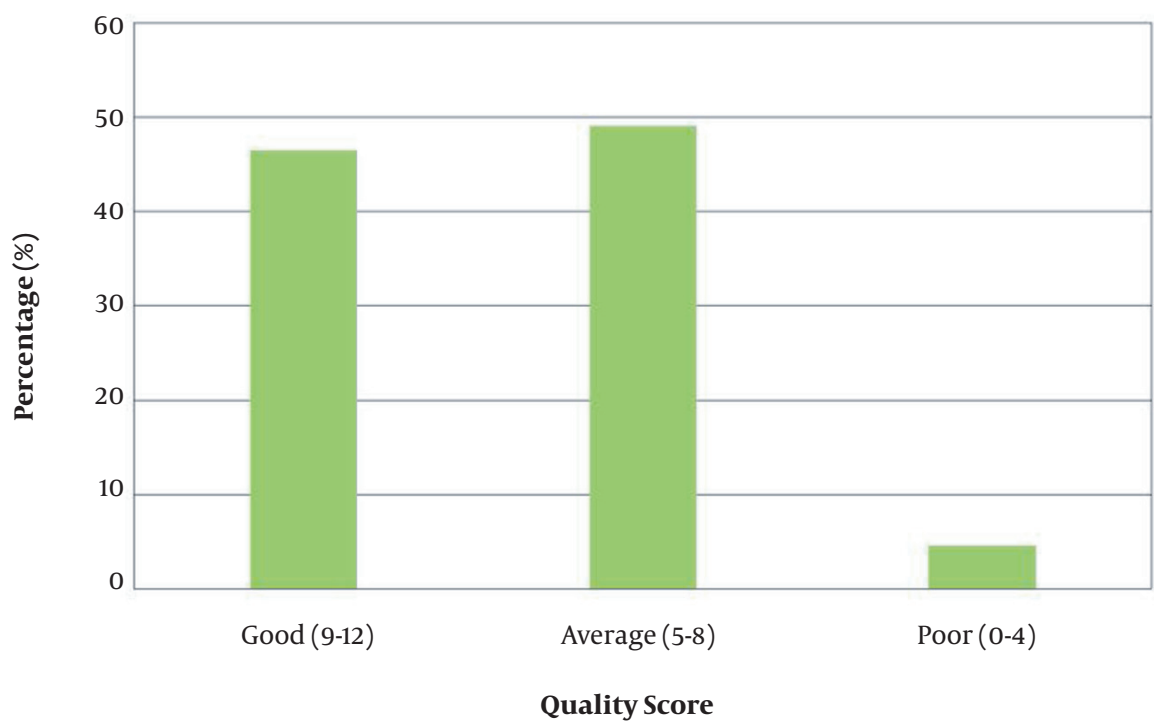

Percentage Distribution of Quality Score

Figure 1. The distribution of the quality scores of audited prescriptions

According to the WHO core prescribing indicators (Table 3), the average number of the medicines prescribed per encounter was 3.5 (ref value $<2$ ), indicating polypharmacy. The percent of the medicines prescribed by generic names was $27.3 \%$ (ref value $100 \%$ ). The ratio of encounters with an antibiotic prescribed was $65 \%$ (ref value $<30 \%$ ), and the percentage of encounters with an injection prescribed was $6 \%$ (ref value $<20 \%$ ). Finally, the rate of prescription from a list of essential medicines was 15\% (ref value 100\%).

Out of 1000 prescriptions, 650 (65\%) had one or more antibiotics prescribed, of which $450(69.2 \%)$ had one antibiotic, 150 (23\%) had two antibiotics, and 50 (7.6\%) had three antibiotics (Figure 2).

Regarding the qualifications of the prescribes of encounters with an antibiotic prescribed, 63\% were postgraduates in pediatrics, 70\% were MBBS physicians, and 90\% were AYUSH doctors (ref value < 30\%).

Out of 1000 prescriptions, antibiotics were prescribed in 650 . The total number of antibiotics used in these prescriptions was 900, indicating the prescription of more than one antibiotic in many sheets. Cephalosporins (443, $44.3 \%$ ) were the most common group of the antibiotics prescribed, followed by penicillin $(228,22.8 \%)$ (Figure 3 ).

\section{Discussion}

In this observational study, we assessed the quality of antibiotic prescribing patterns and prescription writing by child healthcare providers. The quality of the prescriptions was found to be good (46.4\%) and average (49\%) in most encounters, while it was poor at $4.6 \%$. The average number of the medicines prescribed per encounter was 3.5 , indicating polypharmacy. Only $27.3 \%$ of the medicines had been prescribed by their generic names. The percentage of encounters with an antibiotic prescribed was 65\%, indicating a very high antibiotic usage by the health care providers.

Neha et al. (8), in their prescription-audit study involving 100 in-patient prescriptions, found nine prescriptions as semi-rational and 91 as rational. This high rate of rational drug usage was likely to be a result of regular update and supervision by the teaching faculties of the department. In a similar audit study by Ahsan et al. (9) based on WHO core indicators, authors reported the use of 4.02 drugs per prescription with $39.01 \%$ of the prescriptions containing antibiotics. They also reported that $79.2 \%$ of the prescriptions had medications from the list of essential drugs, which was higher than that of our study. This variation in the results of different studies may be explained by different levels of awareness among drug prescribers, the availability of various medicines in a specific area, and differences in the epidemiology of diseases in different regions.

Our study showed that $65 \%$ of the audited prescriptions contained antibiotics. The use of more than one an- 


\begin{tabular}{|c|c|c|}
\hline WHO Core Prescribing Indicators & Reference Value & Observed Value \\
\hline The average number of medicines prescribed per counter & $<2$ & 3.5 \\
\hline The percentage of medicines prescribed by generic names & 100 & 27.3 \\
\hline The percentage of encounters with an antibiotic prescribed & $<30$ & 65 \\
\hline The percentage of encounters with an injection prescribed & $<20$ & 6 \\
\hline The percentage of medicines prescribed from a list of essential medicines & 100 & 15 \\
\hline
\end{tabular}

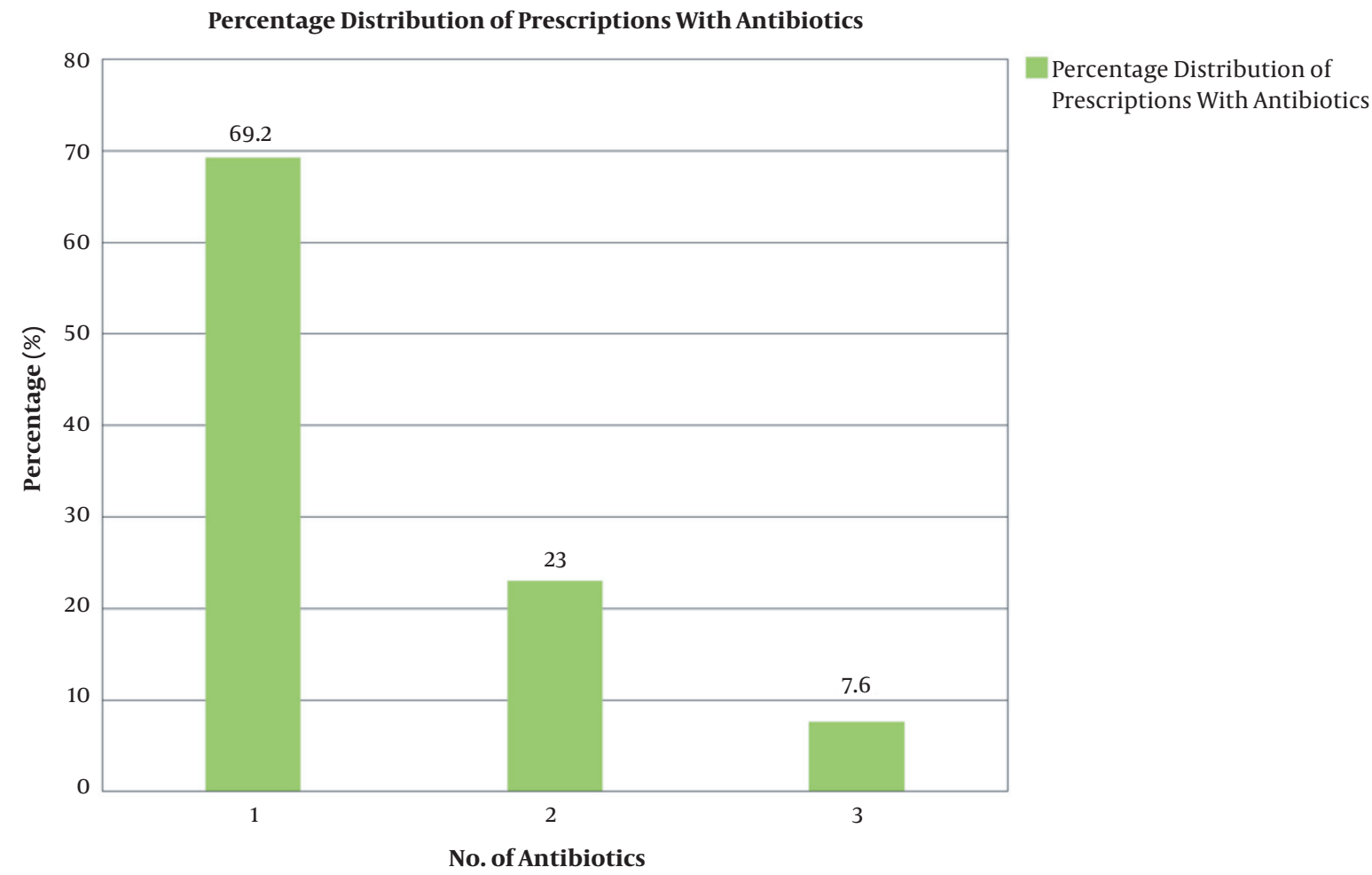

Figure 2. The ratios of prescriptions with one, two, and three antibiotics

tibiotic was relatively common (23 and 7.6\% for two and three antibiotics, respectively). Harshal N Pise et al. (2015), in a study in Maharashtra (10), reported that 60.6\% (110/182) of audited prescriptions contained antibiotics, with most of them containing two antibiotics $(48.58 \%, \mathrm{n}=77 / 182)$. The excessive and simultaneous prescription of more than two antibiotics in a single sheet by outdoor patients necessitates the need for developing and following rational antibiotic prescription policies.

In our study, cephalosporins (44.3\%) were the most common group of the prescribed antibiotics, followed by penicillin (22.8\%). A study by Hussain et al. (11) in rural health care centers showed the use of penicillin in $31.7 \%$ of prescriptions, followed by quinolones and cephalosporins. This difference can be attributed to variations in the populations studied, the number of prescriptions audited, and the prescriber groups' qualifications. Differences in prescribing patterns can also be attributed to variations in the availability of drugs in local markets, as well as the policies of medical representatives.

Many antibiotics in our study were prescribed by MBBS and AYUSH doctors, who are the first point of contact for many sick children. So, it is important to consider training courses, regular updates, and timely feedback by com- 


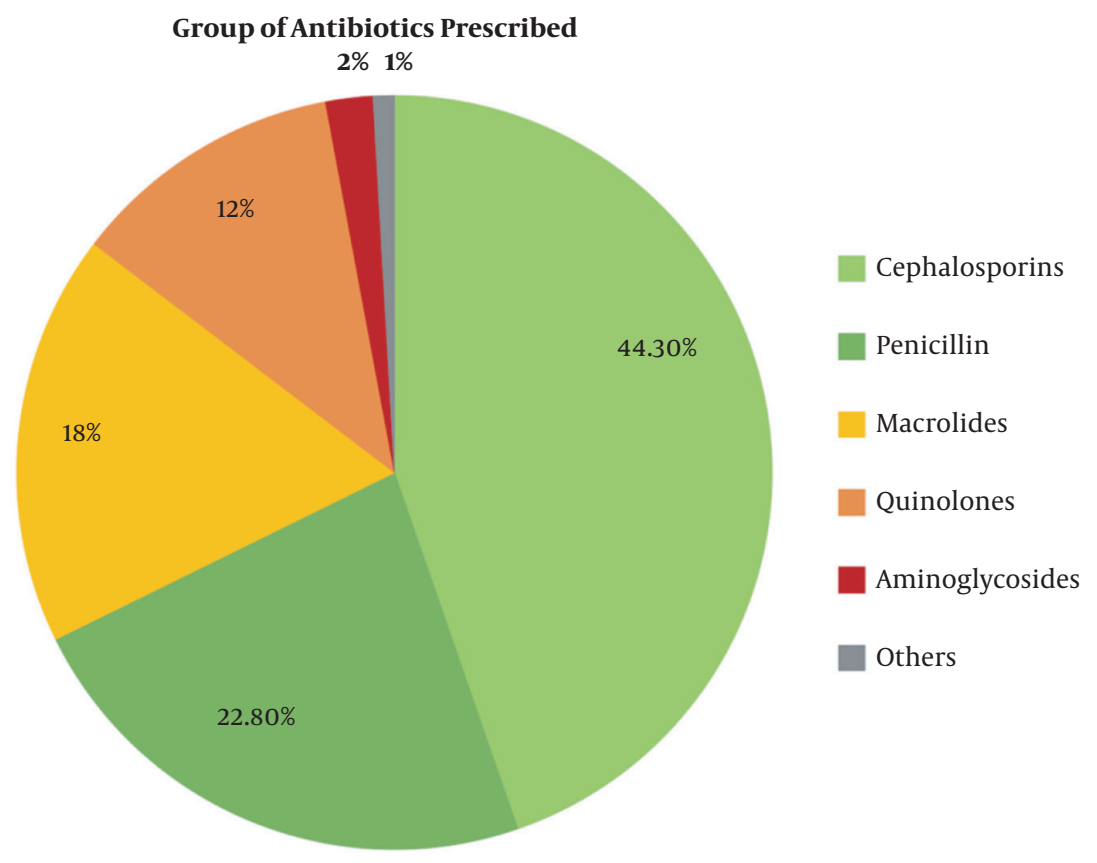

Figure 3. The distribution of various groups of prescribed antibiotics

petent agencies for these practitioners. Our observations also revealed that outpatient training at the postgraduate level was effective in decreasing the indiscriminate use of medicines, especially antibiotics.

The major limitation of our study was that the analysis was confined to a particular geographic region and the duration of the study was only one year. As the study was performed at a single public sector teaching hospital, most of the prescriptions were for the children of the economically poor class of the society.

\subsection{Conclusion}

There is room for the improvement of prescription writing quality. Antibiotic prescription by health care providers, especially AYUSH doctors, needs to be supervised, given that many of these practitioners intend to prescribe antibiotics. Auditing prescriptions over a longer period of time and in different regions will produce betterquality data which can pave the path for formulating effective policies to establish antibiotic stewardship.

\subsection{Recommendations}

- Developing antibiotic stewardship programs at various levels of health care is the need of the hour.
- Periodic prescription audits should be regularly conducted by competent authorities to ensure rational antibiotic use.

\section{Footnotes}

Authors' Contribution: Siva Kumar designed the study protocol, collected the data, and wrote the first draft of the manuscript. Nirbhay Mehta helped in designing the study, provided counseling, and reviewed the manuscript. Prachi Goyal contributed to concept and design, performed data analysis, and approved the manuscript.

Conflict of Interests: There is no conflict of interest.

Ethical Approval: The study was approved by the Institutional Ethics Committee.

Funding/Support: None to declare.

Informed Consent: Informed consent was obtained from participants' parents.

\section{References}

1. Dellinger RP, Levy MM, Rhodes A, Annane D, Gerlach H, Opal SM, et al. Surviving Sepsis Campaign: International guidelines for management of severe sepsis and septic shock, 2012. Intensive Care 
Med. 2013;39(2):165-228. doi: 10.1007/s00134-012-2769-8. [PubMed: 23361625]. [PubMed Central: PMC7095153].

2. Jyoti N, Kaur S. To analyze the impact of serial prescription audits with active feedback on quality of prescription behaviour. J Clin Diagn Res. 2013;7(4):680-3. doi: 10.7860/JCDR/2013/5441.2880. [PubMed: 23730645]. [PubMed Central: PMC3644443].

3. World Health Organization. WHO global strategy for containment of antimicrobial resistance. Geneva, Switzerland: World Health Organization; 2001.

4. Trap B, Hansen EH, Hogerzeil HV. Prescription habits of dispensing and non-dispensing doctors in Zimbabwe. Health Policy Plan. 2002;17(3):288-95. doi: 10.1093/heapol/17.3.288. [PubMed: 12135995].

5. Yin X, Song F, Gong Y, Tu X, Wang Y, Cao S, et al. A systematic review of antibiotic utilization in China. J Antimicrob Chemother. 2013;68(11):2445-52. doi: 10.1093/jac/dkt223. [PubMed: 23800903].

6. World Health Organization. Using indicators to measure country pharmaceutical situations: Fact book on WHO level I and level II monitoring indicators. Geneva, Switzerland: World Health Organization; 2006.
7. Dumoulin J, Kaddar M, Velásquez G; World Health Organization. Guide to drug financing mechanisms. Geneva, Switzerland: World Health Organization; 1998

8. Neha P, Shreya S, Pratik A, Anand A. Prescription auditing and drug utilization pattern in indoor patients of pediatrics department. Int $J$ Cur Res Rev. 2016;8(23):39-46.

9. Ahsan M, Shaifali I, Khurram Mallick A, Kumar Singh HO, Verma S, Shekhar A. Prescription auditing based on World Health Organization (WHO) prescribing indicators in a teaching hospital in North India. Int J Med Res Rev. 2016;4(10):1847-52. doi: 10.17511/ijmrr.2016.110.22.

10. Pise H, Padwal S, Jadhav R, Deshmukh V, Jadhav A, Kolhe A. Drug prescribing and dispensing pattern in pediatrics outpatient clinic of a rural tertiary-care teaching hospital. Natl J Physiol Pharm Pharmacol. 2015;5(4):313-7. doi: 10.5455/njppp.2015.5.0902201546.

11. Hussain S, Parveen Z, Gupta S, Kumar D, Gupta R, Thakur S. A study of prescription auditing in rural health care setting of north India. Int J Med Sci Public Health. 2016;5(12):2461-5. doi: 10.5455/ijmsph.2016.18042016495. 